\title{
ДИАГНОСТИЧЕСКИЕ АСПЕКТЫ УРОВНЯ ФАКТОРОВ POCTA (PDGF-ВВ, ЕРО И НGF) ПРИ РАКЕ ТОЛСТОЙ КИШКИ
}

\section{DIAGNOSTIC ASPECTS OF THE LEVEL OF GROWTH FACTORS (PDGF-BB, EPO AND HGF) IN COLON CANCER}

\section{S. Volkov \\ S. Lobanov \\ A. Yashnov \\ O. Konovalova}

Summary. The article presents the results of a study of the level of growth factors in the blood serum of patients with cancer of the left half of the colon. On average, in cancer patients the level of such comparable substances as EGF, HGF, M-CSF, PDGF-AA, PDGF-BB was several times higher than in the control group $(p<0.05)$. It was also found that at the age of over 50, the level of Angio protein-2, G-CSF, EPO, M-CSF, PDGF$A A, P D G F-B B, V E G F$ is higher than at the age of up to $50(p<0.05)$. The revealed patterns made it possible to create an additive model for the formation of possible risk groups for the development of colon cancer. It was confirmed that the assessment of the level of HGF, EPO, PDGF-BB in blood plasma, in combination with age data, is an additional criterion in the early diagnosis of colon cancer.

Keywords: colon cancer, growth factors, diagnostic markers, test systems, blood tests.

\section{Введение}

$\Pi$ роблемы диагностики рака толстой кишки (РТК) на ранних стадиях связаны с длительным бессимптомным течением опухолевого процесса. Заболеваемость РТК в Российской Федерации в последние годы заметно растёт, более того около 50\% случаев РТК выявляется уже на III-IV стадии болезни, когда появляется отчётливая клиническая картина заболевания (Асcоциация онкологов России, 2019 г.).
Волков Степан Владимирович

Ассистент, ФГБОУ ВО «Читинская государственная медицинская академия» Минздрава России

vsv_19@mail.ru

Лобанов Сергей Леонидович

Д.м.н., профессор, ФГБОУ ВО «Читинская государственная медицинская академия» Минздрава

Poccuu

slobanov15@mail.ru

яинов Алексей Александрович

К.м.н., ассистент, ФГБОУ ВО «Читинская

государственная медицинская академия» Минздрава

Poccuu

alexyashnov@mail.ru

Коновалова Ольга Геннадьевна

К.м.н., дочент, ФГБОУ ВО «Читинская

государственная медицинская академия» Минздрава

Poccuu

konovalovaolgagen@yandex.ru

Аннотация. В статье представлены результаты исследования уровня факторов роста в сыворотке крови больных раком левой половины толстой кишки. Выявлено, что у онкологических пациентов уровень таких сравниваемых веществ как EGF, HGF, M-CSF, PDGF-AA, PDGF-BB был выше, чем В контрольной группе в несколько раз $(p<0,05)$. Так же установлено, что в возрасте старше 50 лет уровень Angio protein-2, G-CSF, EP0, M-CSF, PDGFBB, PDGF-AA, VEGF более высокий, чем в возрасте до 50 лет ( $<<0,05)$. Выявленные закономерности позволили создать аддитивную модель для формирования возможных групп риска по развитию рака толстой кишки. Достоверно подтверждено, что оценка уровня HGF, EPO, PDGF-BB в плазме крови, в сочетании с возрастными данными, является дополнительным критерием в ранней диагностике рака толстой кишки.

Ключевые слова: рак толстой кишки, факторы роста, диагностические маркеры, тест-системы, исследование крови.

Наряду с этим увеличивается удельный вес более молодых пациентов в возрасте до 50 лет [1]. Известно, что опухоли данной локализации, обнаруженные в поздних стадиях, имеют неблагоприятное течение и неудовлетворительные отдалённые результаты. В связи с этим, поиск новых методов ранней диагностики РТК, несомненно, является актуальным.

Использование биологически активных веществ (БАВ) в качестве потенциальных онкомаркеров вызыва- 
Таблица 1. Факторы роста (средние значения, пг / мл)

\begin{tabular}{|l|l|l|l|}
\multirow{2}{*}{ Факторы роста } & Пациенты & \multirow{2}{*}{} \\
\cline { 2 - 4 } & Основная группа & Группа клинического сравнения & \\
\hline Angioprotein-2 & 9,5 & 9,8 & $>0,05$ \\
\hline EGF & 291 & 178,0 & 0,018 \\
\hline EPO & 411 & 147,0 & $>0,05$ \\
\hline FGF-basic & 9,5 & 8,7 & $>0,05$ \\
\hline G-CSF & 23,1 & 15,5 & $>0,05$ \\
\hline GM-CSF & 3,4 & 3,1 & $>0,05$ \\
\hline HGF & 306,4 & 70,4 & $<0,0001$ \\
\hline M-CSF & 114,5 & 56,5 & 0,008 \\
\hline PDGF-AA & 12027,4 & 1251,0 & 0,0004 \\
\hline PDGF-BB & 14329,2 & 4338,1 & 0,022 \\
\hline CSF & 49,7 & 36,8 & $>0,05$ \\
\hline TGF-a & 7,4 & 7,6 & $>0,05$ \\
\hline VEGF & 600,0 & 290,4 & $>0,05$ \\
\hline
\end{tabular}

ет все больший интерес среди учёных и представляется достаточно перспективным. Так в ряде исследований установлено, что уровень некоторых факторов роста (EGF, HGF, TGF- $\beta$, VEGF, PDGF, IP-10, FGF-2, G-CSF, GM-CSF, TNFa и VEGF-A) $[2,3,4,5]$, значительно отличается при РТК относительно контрольной группы. Указанные наблюдения могут свидетельствовать о перспективности использования этих показателей, в качестве вероятных маркеров опухолевого процесса и требуют дальнейшего изучения.

\section{Цель работы}

Определить уровень факторов роста в сыворотке крови больных раком левой половины толстой кишки и оценить возможность использования этих данных в диагностике опухолевого процесса.

\section{Материалы и метолы}

Основная группа включала 63 пациента, оперированных по поводу рака (аденокарциномы) левой половины ободочной кишки (нисходящий, сигмовидный, ректосигмоидный отделы) с I $\mathrm{T}_{1-2} \mathrm{~N}_{0} \mathrm{M}_{0}$, II T $\mathrm{T}_{3-4 a} \mathrm{~N}_{0} \mathrm{M}_{0}$, III $\mathrm{T}_{1-2} \mathrm{~N}_{l} \mathrm{M}_{0}$ стадиями опухолевого процесса. Только у пяти пациентов был выявлен метастаз в одном регионарном лимфоузле. Остальные пациенты были без регионарного метастазирования. У всех пациентов до госпитализации опухоль была подтверждена посредством колоноскопии с последующим гистологическим исследованием. Среди них 32 мужчины и 31 женщина в возрасте от 20 до 75 лет. Средний возраст пациентов составил $57,7 \pm 3,8$ года. Пациенты не получали какого-либо лечения в предоперационном периоде. Группа клинического сравнения в количестве 25 человек составили пациенты с хроническим геморроем вне обострения, которым проводилась колоноскопия. Паци- енты основной и группы клинического сравнения дали добровольное информированное согласие на участие в исследовании.

Забор крови у пациентов основной группы осуществлялся в день операции до ее начала. У пациентов группы клинического сравнения кровь брали после исключения рака толстой кишки (после колоноскопии). Исследование крови осуществлялось с помощью тест-системы (BioLegend): мультиплексный набор для определения факторов роста (Angiopoietin-2, (Ang-2), EGF, EPO, FGF-basic, G-CSF, GM-CSF, HGF, M-CSF, PDGF-AA, PDGF-BB, SCF, TGF-a, VEGF).

\section{Статистика}

Статистическая обработка полученных данных выполнялась при помощи программы Statistica, версии 10.0 .

Проверка нормальности распределения значений переменных в группах наблюдения проводилась с использованием критерия Шапиро-Уилка, распределение принималось нормальным, если р>0,05. Для оценки значимости статистических различий между исследуемыми группами при отсутствии нормального распределения переменных использовали непараметрический ранговый критерий Краскела-Уолиса.

Сила корреляции оценивалась, как статистически значимая, при $p<0,05$. Для анализа корреляционной связи между исследуемыми признакам применялся коэффициент корреляции Пирсона (для нормально распределённых переменных) и коэффициент корреляции Спирмена (если распределение переменных отличалось от нормального). Сила корреляционной связи между признаками оценивалась по коэффициенту r. 
Таблица 2. Уровень исследуемых веществ у пациентов разных возрастных групп

\begin{tabular}{|c|c|c|c|c|c|c|c|}
\hline $\begin{array}{l}\text { Определяемые } \\
\text { параметры }\end{array}$ & Основна & ппа & Групг & иическо & авнения & & \\
\hline Название вещества & $\begin{array}{l}\text { Angio } \\
\text { protein-2 }\end{array}$ & G-CSF & EPO & M-CSF & PDGF-AA & PDGF-BB & VEGF \\
\hline $\begin{array}{l}\text { Уровень вещества (пг/мл) } \\
\text { 50-75 лет }\end{array}$ & 12,7 & 26,4 & 245,6 & 95,2 & 4222,8 & 12074,8 & 536 \\
\hline $\begin{array}{l}\text { Уровень вещества (пг/мл) } \\
\text { 20-49 лет }\end{array}$ & 3,8 & 10,7 & 104,5 & 43,3 & 661,7 & 3310,3 & 234,5 \\
\hline Корреляция Спирмена & 0,037 & 0,06 & $-0,23$ & $-0,314$ & $-0,216$ & $-0,252$ & $-0,25$ \\
\hline Корреляция Гамма & 0,026 & 0,08 & $-0,22$ & $-0,314$ & $-0,213$ & $-0,258$ & $-0,236$ \\
\hline Корреляция Тау Кендалла & 0,025 & 0,08 & $-0,17$ & $-0,242$ & $-0,164$ & $-0,199$ & $-0,182$ \\
\hline$P$ & 0,022 & 0,04 & 0,037 & 0,013 & 0,037 & 0,022 & 0,051 \\
\hline
\end{tabular}

Таблица 3. Составляющие аддитивной модели

\begin{tabular}{|l|l|l|l|l|} 
Предиктор & Коэффициент & ОШ [95\% ДИ] & $\begin{array}{l}\text { Wald } \\
\mathbf{x}^{2}\end{array}$ & $\mathbf{p}$ \\
\hline Константа & $-12,3$ & & 12,87 & 0,000334 \\
\hline Возраст & 0,1639 & $1.18[1.09-1.32]$ & 5,93 & 0,00068 \\
\hline HGF & 0,01944 & $1.02[1.01-1.04]$ & 4,857 & 0,0149 \\
\hline PDGF-BВ & $-0,00045$ & $1[0.999-1]$ & 4,116 & 0,0275 \\
\hline EPO & 0,0239 & $1.02[1.01-1.05]$ & 0,0425 \\
\hline
\end{tabular}

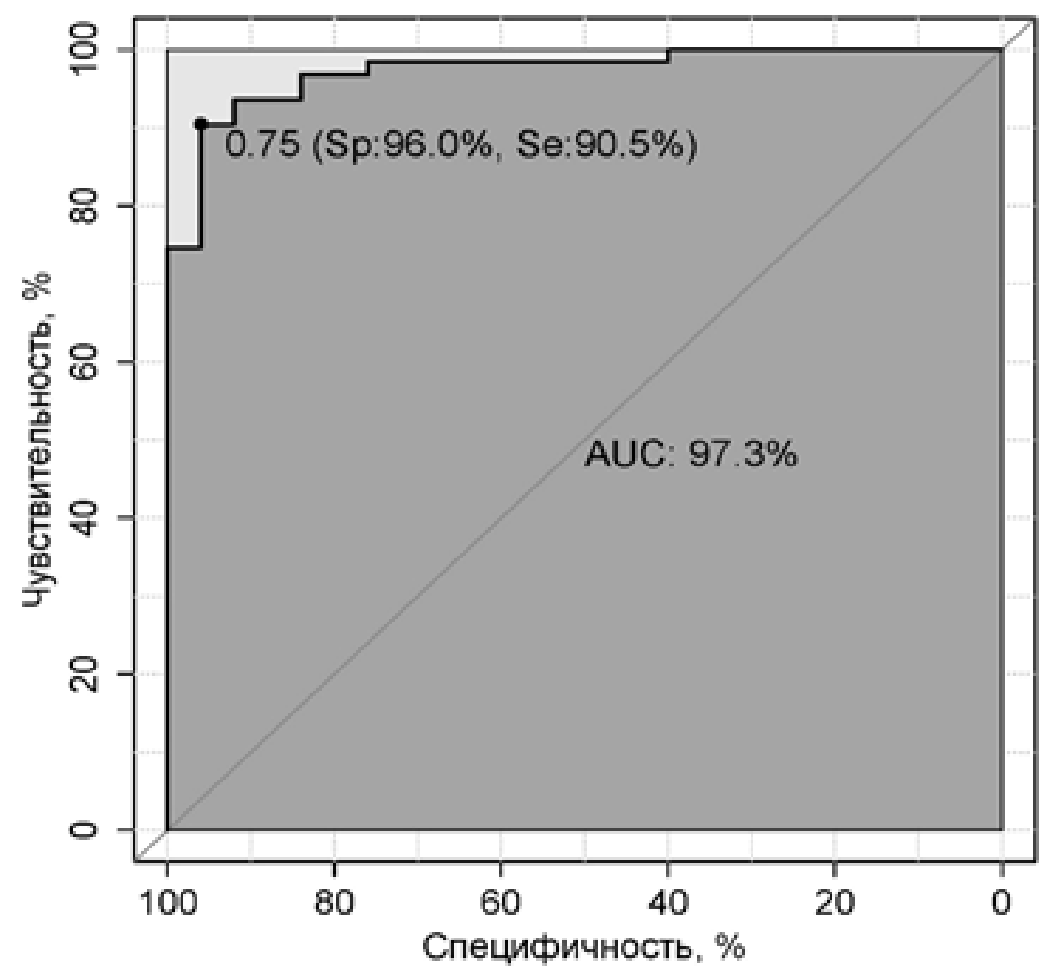

Рис. 1. Результаты ROC анализа аддитивной модели 


\section{Результаты}

В результате сравнения картины крови онкологических больных и группы клинического сравнения, выявлены отличия количественного состава некоторых факторов роста (Таблица 1).

\section{Критерий Краскела-Уолиса (Kruskal-Wallis test)}

В среднем, у онкологических пациентов уровень таких сравниваемых веществ как EGF, HGF, M-CSF, PDGFAA, PDGF-BB был выше, чем в контрольной группе В несколько раз, что является статистически значимым $(\mathrm{p}<0,05)$.

Так же установлена статистически значимая зависимость уровня исследуемых веществ от возраста пациентов. Последние были разделены на до и старше 50 лет, так как эта возрастная граница наиболее чётко показывает различие данных. Выявлено $(p<0,05)$, что в возрасте старше 50 лет уровень Angio protein-2, G-CSF, EPO, M-CSF, PDGF-AA, PDGF-BB, VEGF более высокий, чем в возрасте до 50 лет. Различие статистически значимо (Таблица 2).

При различном сочетании полученных данных, установлена группа веществ (PDGF-BB, EPO и HGF), которая наиболее ярко выражает и подчёркивает выявленные закономерности. Это позволило создать аддитивную модель (чувствительность 96\%, специфичность 90,5\%) для формирования возможных групп риска по развитию рака толстой кишки:

$$
\begin{aligned}
& B=B_{0}+B_{I} * X_{1}+\ldots+B_{n} * X_{n} \\
& B=-12,3+0,1639 * \text { Bозрасm }+0,019 * H G F- \\
& 0,00045 * P D G F-B B+0,024 * E P O \\
& p\left(\text { ИСХОД) }=\frac{1}{1+e^{-B}}\right.
\end{aligned}
$$

При интервале AUC=90-100\% - качество модели отличное; при 80-90\% - хорошее; 70-80\% - среднее; при 60-70\% - слабое и при интервале $50-60 \%$ - неудовлетворительное.

\section{Обсужление}

Известно, что автономность существования опухоли достигается продукцией для себя факторов роста, рецепторов к факторам роста, обеспечивающих свой собственный рост изнутри. Опухолеассоциированные фибробласты считаются наиболее важными элементами клеточного микроокружения опухоли и являются активными участниками канцерогенеза [6]. Последние продуцируют сигнальные белки, в частности HGF, которые стимулируют пролиферацию клеток опухоли [7], экспрессируют факторы ангиогенеза, имеющие особое значение для развития опухоли: PDGF, VEGF [8], что прослеживается и в нашем исследовании.

Подтверждено, что PDGF и HGF способствуют пролиферации и инвазии эпителиальных раковых клеток толстой кишки $[9,10]$. Более того, способность к метастазированию, преимущественно усиливаются с помощью HGF [11]. В одном из исследований оценивали уровень сразу нескольких потенциальных маркеров рака толстой кишки (CCR5, CCL5, PDGF-BB и EphA7). Сделан вывод, что повышенный уровень PDGF-BB может быть использован в ранней диагностике РТК [12].

При этом неоднозначное мнение складывается o EPO. Не однократно подтверждалось, что у пациентов с колоректальным раком уровни ЕРО выше, чем у здоровых людей, но это вполне могло быть связано с гипоксемией, вызванной анемией, обычно присутствующей в этом состоянии [13]. Нами, в настоящем исследовании выявлены статистически значимые отличия уровня PDGF-BB, EPO и HGF в основной и контрольной группах.

Возраст один изважных факторов риска РТК.Появляется все больше данных о росте числа молодых людей в возрасте до 50 лет с РТК[1]. В настоящем исследовании прослеживалось увеличение уровня PDGF-BB и EPO с увеличением возраста пациентов как контрольной, так и основной групп, но статистическая достоверность подтверждена лишь в группе контроля. Этот факт является очередным подтверждением того, что опухолевый процесс находится во взаимосвязи с концентрацией тех или иных биологически активных веществ.

Дополнительно к имеющимся в литературе сведениям [14], нами проведен анализ взаимосвязи клинико-морфологических характеристик опухоли (размер, форма роста), наличия болевого синдрома, опухолевого стеноза и концентрации изучаемых биологически активных веществ. По всем указанным параметрам статистически достоверных отличий не обнаружено. В связи с этим мы сочли возможным не приводить указанные данные в настоящей работе.

Полученные результаты позволяют систематизировать выявленные закономерности в конкретную универсальную аддитивную модель для максимально раннего выявления потенциальной группы риска и последующих диагностических мероприятий.

Таким образом, выявленные корреляционные связи могут быть использованы в клинической практике для ранней диагностики РТК. 


\section{Выво $\triangle \mathrm{b}$}

1. установлено, что уровень содержания HGF, PDGFВВ в плазме крови больных РТК достоверно отличается от таковых в группе людей с неопухолевой патологией, а ЕРО выше у пациентов старше 50 лет в обоих группах.
2. созданная аддитивная модель может быть использована в качестве одного из критериев в комплексной диагностике раннего рака толстой кишки, что позволит оптимизировать лечебную тактику и использовать хирургический метод лечения РТК в качестве основного и единственного.

\section{ЛИТЕРАТУРА}

1. Weinberg B, Marshall J. Colon Cancer in Young Adults: Trends and Their Implications. Curr Oncol Rep. 2019 Jan 18;21(1):3. doi: 10.1007/s11912-019-0756-8

2. Üçüncü M, Serilmez M, Sarı M, Bademler S, Karabulut S. The diagnostic significance of PDGF, EphA7, CCR5, and CCL5 Levels in Colorectal Cancer. Biomolecules. 2019 Sep 9;9(9). pii: E464. doi: 10.3390/biom9090464.

3. Krzystek-Korpacka M, Zawadzki M, Kapturkiewicz B, Lewandowska P, Bednarz-Misa I, Gorska S, Witkiewicz W, Gamian A. Subsite heterogeneity in the profiles of circulating cytokines in colorectal cancer. Cytokine. 2018 0ct;110:435-441. doi: 10.1016/j.cyto.2018.05.015.

4. Lian G, Chen S, Ouyang M, Li F, Chen L, Yang J. Colon cancer cell secretes EGF to promote M2 polarization of TAM through EGFR/PI3K/AKT/mTOR pathway. Technol Cancer Res Treat. 2019 Jan 1;18:1533033819849068. doi: 10.1177/1533033819849068.

5. Herrera A, Herrera M, Guerra-Perez N, Galindo-Pumariño C, Larriba MJ, García-Barberán V, Gil B, Giménez-Moyano S, Ferreiro-Monteagudo R, Veguillas $P$, Candia $A$, Peña R, Pinto J, García-Bermejo ML, Muñoz A, García de Herreros A, Bonilla F, Carrato A, Peña C. Endothelial cell activation on 3D-matrices derived from PDGF-BBstimulated fibroblasts is mediated by Snail1. Oncogenesis. 2018 Sep 24;7(9):76. doi: 10.1038/s41389-018-0085-Z.

6. Зибиров Р.Ф., Мозеров С.А. Характеристика клеточного микроокружения опухоли. Онкология. Журнал Им. П.А. Герцена. 2018;2:67-72. URL : https://www. mediasphera.ru/issues/onkologiya-zhurnal-im-p-a-gertsena/2018/2/12305218X2018021067/annotation

7. Cirri P, Chiarugi P. Cancer-associated-broblasts and tumour cells: a diabolic liaison driving cancer progression. Cancer Metastasis Rev. 2012;31(1-2):195-208. https:// doi.org/10.1007/s10555-011-9340-x

8. Kamińska K, SzczylikC, Bielecka ZF, Bartnik E, Porta C, Lian F, Czarnecka AM. The role of the cell-cell interactions in cancer progression. J Cell Mol Med. 2015;19(2):283296. https://doi.org/10.1111/jcmm.12408

9. Li F, Zhu Y-T. HGF-activated colonic fibroblasts mediates carcinogenesis of colonic epithelial cancer cells via PKC-cMET-ERK1/2-COX-2 signaling. Cell Signal. 2015 Apr;27(4):860-6. doi: 10.1016/j.cellsig.2015.01.014

10. Moench R, Grimmig T, Kannen Y, Tripathi S, Faber M, Chandraker A, Lissner R, Germer K-T, Maria Waaga-Gasser A, Gasser M. Exclusive inhibition of PI3K/Akt/mTOR signaling is not sufficient to prevent PDGF-mediated effects on glycolysis and proliferation in colorectal cancer. Oncotarget. 2016 0ct 18;7(42):68749-68767. doi: 10.18632/oncotarget.11899

11. Yao J-F, Li X-J, Yan L-K, He S, Zheng J-B, Wang X-R, Zhou P-H, Zhang L. Role of HGF/c-Met in the treatment of colorectal cancer with liver metastasis. J Biochem Mol Toxicol. 2019 Jun;33(6):e22316. doi: 10.1002/jbt.22316

12. Üçüncü M, Serilmez M, Sarı M, Bademler S, Karabulut S. The Diagnostic Significance of PDGF, EphA7, CCR5, and CCL5 Levels in Colorectal Cancer. Biomolecules. 2019 Sep 9;9(9):464. doi: 10.3390/biom9090464

13. Bessler H, Herman-Edelstein M, Djaldetti M. About the interconnection between Erythropoietin, Mononuclears and colon carcinoma cells. International Journal of Hematology and Blood Disorders. 2018 0ct 9;9(9):464. doi: 10.15226/2639-7986/3/3/00126

14. Sinha A, Kumar S. Prognostic value of Epidermal growth factor receptor in colorectal carcinoma. Journal of Clinical and Diagnostic Research. 2018 March; 12(3):1-4. doi:10.7860/JCDR/2018/34864.11234.

(c) Волков Степан Владимирович ( vsv_19@mail.ru ), Лобанов Сергей Леонидович ( slobanov15@mail.ru ), Яшнов Алексей Александрович ( alexyashnov@mail.ru ), Коновалова Ольга Геннадьевна ( konovalovaolgagen@yandex.ru ). Журнал «Современная наука: актуальные проблемы теории и практики» 